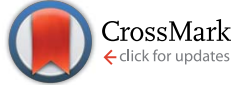

Cite this: J. Mater. Chem. C, 2015, 3, 2237

Received 21st November 2014

Accepted 11th January 2015

DOI: $10.1039 / \mathrm{c} 4 \mathrm{tc0} 2660 \mathrm{~h}$

www.rsc.org/MaterialsC

\section{High density array of multiferroic nanoislands in a large area†}

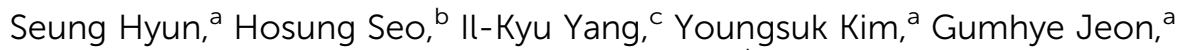 \\ Bom-yi Lee, ${ }^{a}$ Yoon Hee Jeong, ${ }^{C}$ Yunseok Kim ${ }^{\star b}$ and Jin Kon Kim ${ }^{\star a}$
}

\begin{abstract}
We report the innovative fabrication of a high density array of multiferroic $\mathrm{BiFeO}_{3}$ (BFO) nanoislands on a conductive substrate in a large area. The anodic aluminum oxide (AAO) template has widely been used to fabricate highly arranged nanostructures, because of easy control of pore size and perfect hexagonal pore packing. The existing AAO mask-assisted pulsed laser deposition (PLD) method is limited to fabricating a nanoisland array in a small area. To supplement the shortcoming of this method, a thick AAO membrane in a large area was electrochemically detached and floated on polystyrene (PS) film without crack. Then, a nanoporous polystyrene (PS) template was prepared by dry etching with the thick AAO membrane mask, followed by spin coating of the BFO precursor on the PS template. After removing the PS template at high temperature, we prepared a high density array of multiferroic BFO nanoislands in a large area epitaxially grown on an STO:Nb (100) substrate. A high density array of BFO nanoislands in a large area showed both ferroelectricity of individual nanoislands obtained by piezoresponse force microscopy (PFM) and macroscopic magnetism measured by a superconducting quantum interference device (SQUID) based magnetic property measurement system (MPMS). A high density array of BFO nanoislands could be employed as a next-generation memory device capable of electric writing and magnetic reading (or vice versa).
\end{abstract}

\section{Introduction}

Multiferroics with ferroelectric, (anti-)ferromagnetic and ferroelastic properties have generated a strong interest for nextgeneration storage materials because of their novel and strong coupling effect between the three properties. Among many multiferroic materials, $\mathrm{BiFeO}_{3}(\mathrm{BFO})$ is particularly promising because it exhibits both ferroelectricity and magnetism at room temperature. ${ }^{1-8}$ When multiferroic $\mathrm{BFO}$ is utilized, electric writing followed by magnetic reading (or magnetic writing followed by electric reading) is possible, which is essential for developing next-generation memory devices. ${ }^{1,2}$ Writing data controlled by voltage has three advantages: (i) it could be used in solid circuits, (ii) very thin devices are possible, and (iii) voltage is reduced, which saves energy. Reading data by a magnetic field is a non-destructive method. ${ }^{1,2}$

${ }^{a}$ National Creative Research Center for Smart Block Copolymers, Department of Chemical Engineering, Pohang University of Science and Technology (POSTECH), San 31 Hyoja-dong, Pohang 790-784, Republic of Korea. E-mail: jkkim@postech.ac.kr ${ }^{5}$ School of Advanced Materials Science and Engineering, Sungkyunkwan University, Suwon, Gyeonggi-do 440-746, Republic of Korea. E-mail: yunseokkim@skku.edu 'Department of Physics and Electron Spin Science Center, Pohang University of Science and Technology, Pohang 790-784, Republic of Korea

$\dagger$ Electronic supplementary information (ESI) available. See DOI: $10.1039 / \mathrm{c} 4 \mathrm{tc} 02660 \mathrm{~h}$
Although continuous multiferroic BFO thin film has shown the possibility for various applications such as information storage media, ${ }^{1-3}$ discrete nanostructures such as nanoislands are ideal because an individual nanoisland acts as its own memory unit. Furthermore, the switching state of the BFO nanoisland is much more stable than BFO thin film because thin film is very sensitive to undesirable domain switching due to the mechanical cross-talk effect. ${ }^{2}$

Several methods for preparing the array of BFO nanoislands have been introduced in the literature: soft electron beam lithography (soft-eBL), ${ }^{9}$ dip-pen lithography, ${ }^{10,11}$ block copolymer template, ${ }^{12,13}$ self-assembled growth, ${ }^{14}$ ion beam milling, ${ }^{15}$ and anodic aluminum oxide (AAO) template.$^{16-19}$ Because softeBL requires that the electron beam must be scanned across patterned areas pixel by pixel, it needs long times and requires an expensive and highly sophisticated machine. Similarly, dippen lithography takes a long time to fabricate a high density array in a large area. Self-assembled growth is hard for controlling the size of the nanoisland and fabricating wellarranged nanoislands in a large area. Also, ion beam milling uses high energy irradiation during etching, which could damage the crystal structure of nanoislands.

Recently, Hong et al. used an AAO template to fabricate a high density array of ferroelectric nanoislands on a solid conducting surface and showed that individual BFO nanoislands showed ferroelectric switching. ${ }^{16}$ However, due to the very poor 
contact (or adhesion) between the AAO membrane and the conducting substrate, the pulsed laser deposition (PLD) method is only used to prepare a high density array of multiferroic islands in a small area. In this situation, a very thin AAO membrane should be used, which limits control of the height of BFO nanoislands on a solid substrate. Also, it is not easy to achieve uniform contact with the substrate in a large area; thus the lateral area of the nanoisland array is limited (a range of $\sim \mathrm{mm}^{2}$ ).

Wang et al. reported that BFO thin film with a thickness of 70 $\mathrm{nm}$ on STO:Nb (100) showed large saturation magnetization compared with bulk BFO because of the mismatched strain between BFO and STO:Nb. ${ }^{4}$ However, there is no report on magnetic properties of individual BFO nanoislands in a high density array.

Therefore, it is utmost necessary to find a facile method to fabricate a high density array of multiferroic BFO nanoislands in a large area (for example, a wafer size), and investigate both ferroelectric and magnetic properties of individual BFO nanoislands.

To achieve the above objective, one should use spin-coating of multiferroic precursors in solution, followed by a sol-gel method. In this case, an excellent contact (or good adhesion) between the nanoporous template and solid substrate is needed, because some areas of the AAO membrane are peeled off from the substrate during spin-coating (or wet process). We realized that once a nanoporous polymer template was used instead of the AAO membrane, the contact between polymer template and a conducting substrate was very excellent. ${ }^{20}$ This is easily employed for a wet process such as the sol-gel method.

In this study, we used a nanoporous polystyrene (PS) template where the pores were prepared by reactive-ion etching with the aid of the AAO membrane. Because of excellent adhesion between polymer film and the substrate, BFO precursors are easily incorporated into nanopores in the polymer template by spin-coating in a large area. Due to the existence of a PS matrix and the direct contact with the substrate, each BFO nanoisland in each nanopore could not be agglomerated with each other even after thermal treatment for the removal of the organic moieties in the BFO precursor as well as the PS matrix. Because a nanoisland array was formed through the entire area, the BFO crystals were characterized by synchrotron X-ray diffraction.

The high density array of BFO nanoislands in a large area exhibited both ferroelectricity of individual nanoislands observed by piezoresponse force microscopy (PFM) and macroscopic magnetism measured by a superconducting quantum interference device (SQUID) based magnetic property measurement system (MPMS). Also, we achieved simultaneous switching of ferroelectricity of individual nanoislands and macroscopic magnetism. Furthermore, the high density array of BFO nanoislands exhibited unexpectedly large magnetization compared with BFO thin film. A high density array of BFO nanoislands could be employed as a next-generation memory device capable of electric writing and magnetic reading (or vice versa).

\section{Experimental section}

\section{Preparation of polystyrene film}

$4 \mathrm{wt} \%$ of polystyrene (PS, $M_{\mathrm{n}}: 103$ 700, PDI: 1.0, Polymer Source) in toluene (AR grade) was spin-coated at a rotating speed of 6000 rpm for $60 \mathrm{~s}$ on thermally treated STO:Nb (100). The film thickness measured by ellipsometry was $136 \mathrm{~nm}$.

\section{Preparation of AAO template}

The anodic aluminum oxide (AAO) membrane was prepared by two-step anodization. A highly pure aluminum plate (99.999\%, $1 \mathrm{~mm}$ thickness) was sonicated in acetone for $30 \mathrm{~min}$ and electrochemically polished twice in a polishing solution (ethanol : perchloric acid $=4: 1 \mathrm{v} / \mathrm{v}$ ) at $7{ }^{\circ} \mathrm{C}$ and $20 \mathrm{~V}$ for $30 \mathrm{~min}$. Then, a polished aluminum plate was washed with ethanol and DI-water alternately. The first anodization was carried out in oxalic acid $(0.3 \mathrm{M})$ at $15{ }^{\circ} \mathrm{C}$ and $40 \mathrm{~V}$ for $12 \mathrm{~h}$, and was etched by an etchant solution (chromium oxide: $1.8 \mathrm{wt} \%$ and $85 \%$ phosphoric acid: $6 \mathrm{wt} \%$ in DI-water) at $65^{\circ} \mathrm{C}$ for $5 \mathrm{~h}$, and washed with DI-water. The second anodization was performed at $15{ }^{\circ} \mathrm{C}$ and $40 \mathrm{~V}$ for $10 \mathrm{~min}$ to obtain hexagonally packed cylindrical nanopores with a diameter of $20 \mathrm{~nm}$. The thickness of the porous membrane was $1 \mu \mathrm{m}$. Finally, to widen the pore size, the porous AAO template was dipped into $0.1 \mathrm{M} \mathrm{H}_{3} \mathrm{PO}_{4}$ aqueous solution at $30{ }^{\circ} \mathrm{C}$ for $50 \mathrm{~min}$.

\section{Fabrication of a high density array of BFO nanoislands}

First, the BFO precursor was prepared by dissolving $8.00 \mathrm{~g}$ of bismuth nitrate $\left[\mathrm{Bi}\left(\mathrm{NO}_{3}\right)_{3} \cdot 5 \mathrm{H}_{2} \mathrm{O}\right]$ and $6.06 \mathrm{~g}$ of iron nitrate $\left[\mathrm{Fe}\left(\mathrm{NO}_{3}\right)_{3} \cdot 9 \mathrm{H}_{2} \mathrm{O}\right]$ into $20 \mathrm{ml}$ of 2-methoxyethanol for $30 \mathrm{~min}$ at room temperature. The mole ratio of bismuth nitrate to iron nitrate was 1.1 because a small amount of Bi was lost at high temperature. Then, $10 \mathrm{ml}$ of acetic anhydride was added followed by $0.1 \mathrm{ml}$ of ethanolamine to adjust the viscosity and stirred for $60 \mathrm{~min}$. The concentration of the stock solution was adjusted to $0.3 \mathrm{M}$ by adding 2-methoxyethanol under stirring. Finally, the concentration of BFO precursor solution for spincoating was decreased to $0.01 \mathrm{M}$, after addition of glacial acetic acid to the solution. The BFO precursor was dropped on the PS template and then spin-coated at $4000 \mathrm{rpm}$ for $60 \mathrm{~s}$. The precursor was heated at $300{ }^{\circ} \mathrm{C}$ and maintained at this temperature for $15 \mathrm{~min}$ to remove the PS template and the organic moiety in the precursor. Then, the temperature was raised to $600{ }^{\circ} \mathrm{C}$ and maintained for $1 \mathrm{~h}$. During this process, BFO nanoislands were epitaxially grown on the single crystalline STO:Nb (100) substrate.

\section{Characterizations}

Surface topography of the BFO nanoislands was observed by scanning electron microscopy (SEM, Hitachi, S4800) and atomic force microscopy (AFM, Digital Instrument) with tapping mode. Qualitative and quantitative analyses were conducted by X-ray photoelectron spectroscopy (XPS, EscaLab 220-IXL). The crystal structure of BFO nanoislands was characterized by synchrotron X-ray diffraction (XRD, Pohang Accelerator Laboratory, 3D 
beamlines, $E=8.05011 \mathrm{keV}, \lambda=1.54016 \AA$ ). High-resolution transmission electron microscopy (HRTEM, JEOL, JEM-2100F) was used to observe the cross-sectional image of a single nanoisland. Piezoresponse force microscopy (PFM) was performed to observe piezoresponse properties of individual BFO nanoislands by AFM (NX10, Park Systems) combined with a lock-in amplifier (SR830, Stanford Research Systems). An AC voltage of $0.5 \mathrm{~V}$ at a frequency of $17 \mathrm{kHz}$ was applied to a conductive probe (Multi75E-G, BudgetSensors®). A superconducting quantum interference device (SQUID) based magnetic property measurement system (MPMS XL-7, Quantum Design) was used to measure magnetic property macroscopically.

\section{Results and discussion}

Fig. 1 is a schematic of the fabrication of a high density array of BFO nanoislands on a conducting substrate. First, polystyrene (PS) thin film was prepared by spin coating on a reconstructed STO:Nb (100) substrate by pre-annealing at $1000{ }^{\circ} \mathrm{C}$ for $2 \mathrm{~h} \cdot .^{12,13,21}$ (step a) (ESI Fig. S1 and S2 $\dagger$ ). An AAO template fabricated by two-step anodization was placed on PS film (step b). Incomplete and unopened pores located at the backside of the AAO template are fully opened by $\mathrm{CF}_{4}$ reactive ion etching (step c). Then, using $\mathrm{O}_{2}$ reactive ion etching with the aid of an AAO mask followed by the detachment of the AAO membrane from the PS template by $3 \mathrm{M}$ Scotch tape, the porous PS template was obtained (step d). The BFO precursor was dropped into the nanoporous PS template and spin coated (step e). Finally, we obtained a high density array of BFO nanoislands in a large area after removal of the PS template as well as the organic moieties in BFO precursors by combustion at high temperature (step f). During this process, BFO nanoislands were epitaxy-crystallized

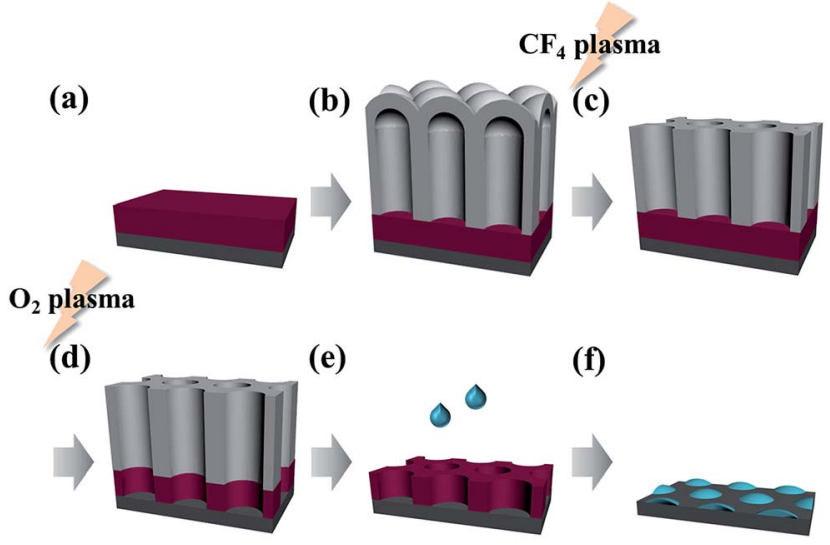

Fig. 1 Schematic flow chart for the fabrication procedure of high density BFO nanoislands. (a) Polymer thin film spin coated on the reconstructed STO:Nb (100) substrate. (b) The AAO template is placed on polymer thin film. (c) The incompletely opened AAO template is etched by $\mathrm{CF}_{4}$ reactive etching. (d) The porous polymer template was fabricated by $\mathrm{O}_{2}$ reactive etching based on an AAO mask and then AAO was detached using 3M tape. (e) The BFO precursor was dropped and then spin coated. (f) High density BFO nanoislands over a large area after heating at high temperature. on the substrate. The detailed experimental procedure is given in section 1 of the ESI. $\dagger$

Fig. 2 shows the fabrication of the nanoporous polystyrene (PS) template on a solid substrate. First, the AAO template with a pore diameter of $60 \mathrm{~nm}$ was prepared by a two-step anodization process. The pore size of the AAO template was easily controlled using different kinds of electrolytes and widening time. Thus, various sizes of nanoislands could be fabricated by changing the pore size of the AAO template (ESI Fig. S2†). The AAO template was electrochemically detached from aluminum by applying $55 \mathrm{~V}$ at $7{ }^{\circ} \mathrm{C}$ for $3 \mathrm{~s}$ and floated on PS film in ethanol solution. The open side of the AAO template was placed on the PS film (Fig. 2a). When an AAO template with a large area (for instance, $5 \mathrm{~cm} \times 5 \mathrm{~cm}$ : see Fig. S3a $\dagger$ ) is detached from the $\mathrm{Al}$ substrate and floated on PS film, it is very important to use a thick AAO template to have uniform coverage. For instance, when an AAO template with a thickness of at least $1 \mu \mathrm{m}$ was used, it was successfully floated on PS thin film throughout the entire area. But, if a thin AAO template (a thickness of $300 \mathrm{~nm}$ ) was detached from the Al substrate and floated on PS film, only small portions of the AAO template were floated on PS film because of tearing and resulting from the bubbles formed during the electrochemical detachment process (ESI Fig. S3†).

Fig. 2b shows the electrochemically detached AAO template. Many pores at the top of the AAO were closed. To obtain open pores, $\mathrm{CF}_{4}$ plasma etching was performed using an inductive coupled plasma etcher for $5 \mathrm{~min}$ (a flow of $5 \mathrm{sccm}$, a working pressure of 10 mTorr, a chuck power of $20 \mathrm{~W}$, and a source power of $50 \mathrm{~W}$ ) as shown in Fig. 2c. Then, a nanoporous PS template was prepared by $\mathrm{O}_{2}$ plasma etching for $3 \mathrm{~min}$ with the thick AAO membrane as an etch mask, as shown in Fig. 2d. The conditions for $\mathrm{O}_{2}$ plasma etching were a flow of $5 \mathrm{sccm}$, a working pressure of 10 mTorr, a chuck power of $0 \mathrm{~W}$, and a source power of $80 \mathrm{~W}$. Because of the sharp edge of AAO, the pore size of the PS template is $63 \mathrm{~nm}$, which is slightly larger than that $(60 \mathrm{~nm})$ of the AAO template. Finally, the AAO mask on the polystyrene template was detached by peeling off with 3 $\mathrm{M}$ tape because of a poor adhesion between the AAO template and PS as shown in Fig. 2e.

We prepared the PS template with a $63 \mathrm{~nm}$ pore size on an STO:Nb (100) conductive substrate. Fig. $3 \mathrm{a}$ and b give the topview and $30^{\circ}$ tilted view by field emission scanning electron microscopy (FE-SEM). BFO nanoislands are well ordered in a large area (wafer scale). To find the size distribution of BFO nanoislands in the array, we obtained the topographic image using atomic force microscopy (AFM) (Fig. 3c). From the histogram shown in Fig. 3d and e, the average diameter and the height of BFO nanoislands in the array were $47 \pm 10$ and $15 \pm 5$ $\mathrm{nm}$, respectively. Thus, the aspect ratio (height/diameter) is 0.32 .

To check whether any impurity exists in the BFO nanoislands, we performed X-ray photoelectron spectroscopy (XPS).

Fig. $4 \mathrm{a}$ shows that the Bi $4 \mathrm{f}$ peak consists of two peaks: $4 \mathrm{f} 7 / 2$ $(158.9 \mathrm{eV})$ and $4 \mathrm{f} 5 / 2(164.2 \mathrm{eV})$. Since spin orbital splitting energy in pure $\mathrm{Bi} 4 \mathrm{f}$ is $5.3 \mathrm{eV}(164.2 \mathrm{eV}$ to $158.9 \mathrm{eV})$, it is well matched with the theoretical value $(5.36 \mathrm{eV}) .{ }^{22-24}$ For the $\mathrm{Fe}^{3+} 2 \mathrm{p}$ peak in Fig. 4b, 2p 3/2 (711.1 eV) and 2p 1/2 (724.4 eV) are 
(a)

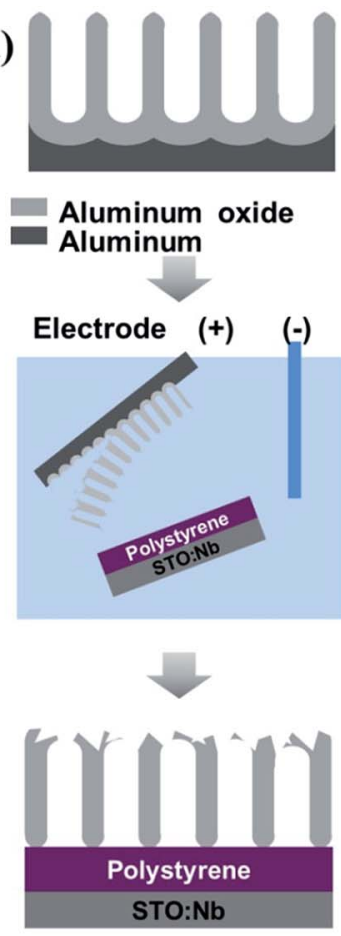

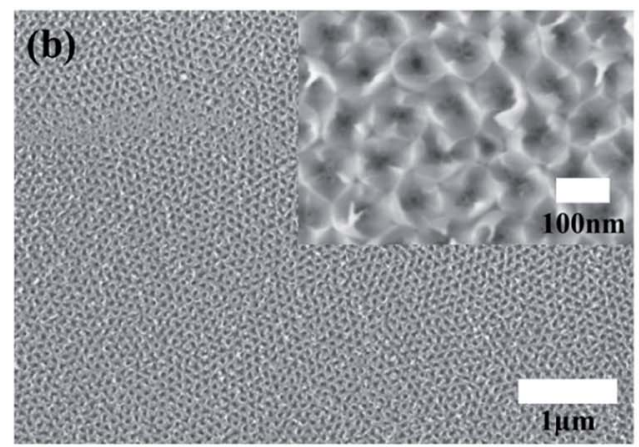

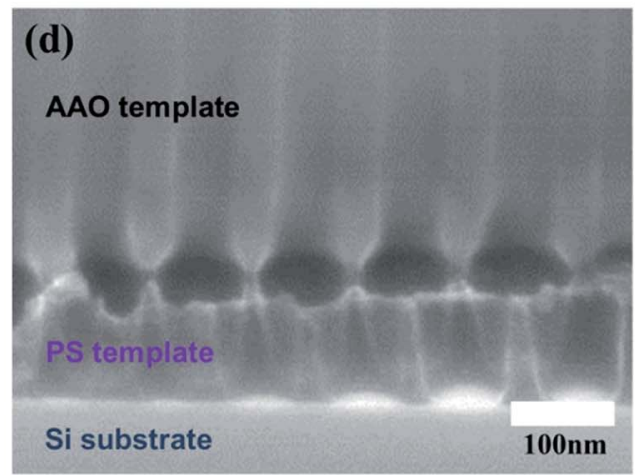

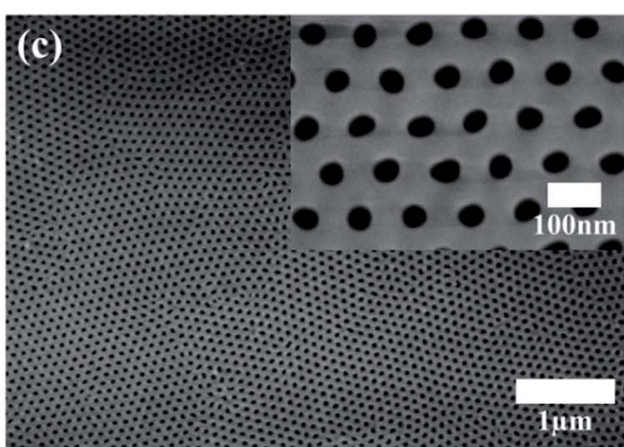

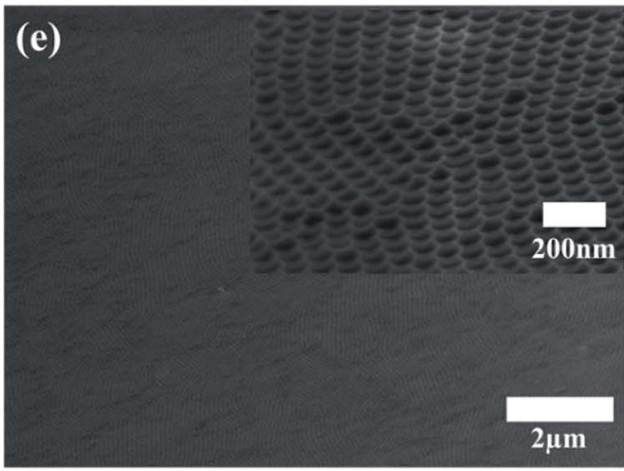

Fig. 2 (a) Experimental scheme of electrochemically detached AAO template and floating on PS film. (b) Top view SEM image of the unopened AAO template. Inset: magnified view. (c) Top view SEM image of the opened AAO template by $\mathrm{CF}_{4}$ plasma etching. Inset: a magnified view. (d) Cross-sectional SEM image of a nanoporous PS template prepared by $\mathrm{O}_{2}$ plasma etching using the AAO template. (e) Top view of the nanoporous PS template after removal of the AAO template. Inset: a magnified view.
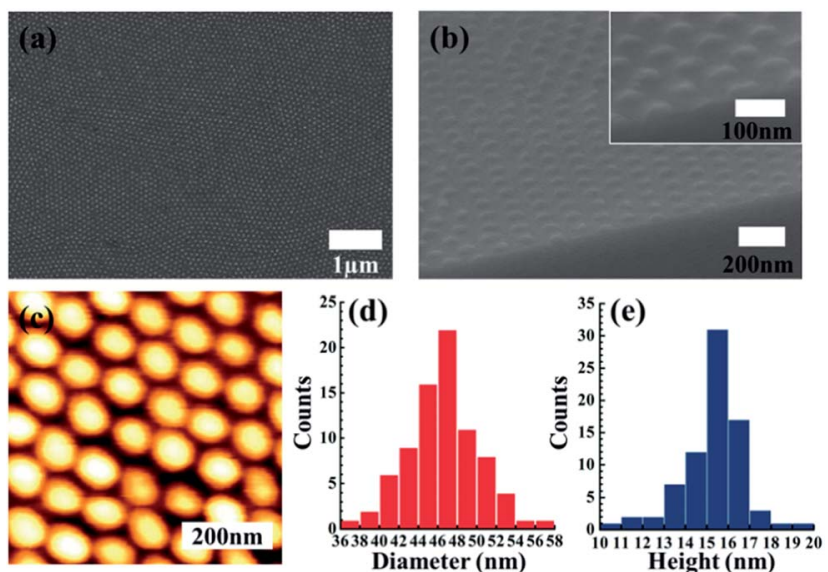

Fig. 3 (a) Plane view (a) and $30^{\circ}$ tilted SEM images of the array of BFO nanoislands. (c) AFM topography image of the array of BFO nanoislands. Histograms of (d) the diameter and (e) the height of BFO nanoislands measured by AFM topography image including 200 BFO nanoislands.
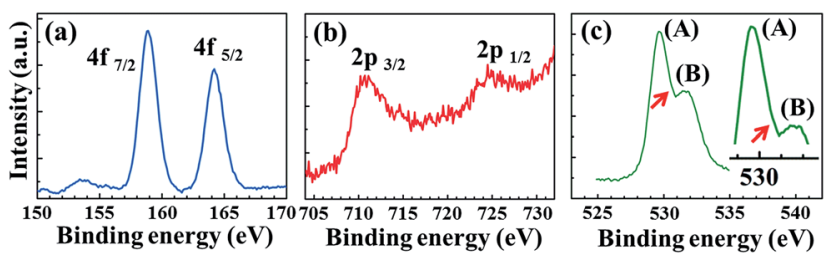

Fig. 4 XPS analysis of BFO nanoislands (a) Bi $4 f$ line (b) Fe $2 p$ line and (c) $\mathrm{O}$ 1s line. The inset in (c) shows a magnified view near $530 \mathrm{eV}$. observed. The theoretical spin orbit splitting energy of the Fe $2 p$ doublet is $13.6 \mathrm{eV}$, which is almost the same as the experimental value $(13.4 \mathrm{eV})$. Since there is no peak at $708 \mathrm{eV}$, which corresponds to $\mathrm{Fe}^{2+}$ 2p $3 / 2,{ }^{22-24} \mathrm{BFO}$ islands in this experiment contain only $\mathrm{Fe}^{3+}$, not $\mathrm{Fe}^{2+}$. However, the existence of only $\mathrm{Fe}^{3+}$ could not confirm that the sample consists of pure $\mathrm{BFO}$ without any second phase (for instance, $\gamma-\mathrm{Fe}_{2} \mathrm{O}_{3}$ which has been well known as a ferromagnetic material).

This is because the iron in $\gamma-\mathrm{Fe}_{2} \mathrm{O}_{3}$ is also $\mathrm{Fe}^{3+}$. Thus, we obtained an XPS spectrum of oxygen 1s peaks. As shown in Fig. 4c, a peak (A) at $529.6 \mathrm{eV}$ represents $\mathrm{O} 1 \mathrm{~s}$ in pure $\mathrm{BFO}$, while another peak (B) at $531.5 \mathrm{eV}$ comes from the chemisorbed oxygen species. ${ }^{25}$ A shoulder (marked by the arrow) near 530.5 $\mathrm{eV}$ comes from O 1s in either the STO:Nb substrate or $\gamma-\mathrm{Fe}_{2} \mathrm{O}_{3}$. To clarify whether $\gamma-\mathrm{Fe}_{2} \mathrm{O}_{3}$ indeed exists in the sample, we carried out synchrotron X-ray diffraction (XRD) covering $2 \theta$ from $15^{\circ}$ to $75^{\circ}$. XRD patterns were successfully obtained because the array of BFO nanoislands was prepared in a large area. Fig. 5a shows a $\theta-2 \theta$ scan along the out-of-plane to the substrate. BFO (001) and (002) peaks are clearly observed near the STO:Nb (001) and (002) peaks, respectively. Although distinct diffraction peaks (marked by blue dots) belonging to $\gamma-\mathrm{Fe}_{2} \mathrm{O}_{3}$ crystals are not observed, a broad and very weak peak is seen at $2 \theta \sim 30^{\circ}$. This indicates that it might be possible that $\gamma-\mathrm{Fe}_{2} \mathrm{O}_{3}$ exists in $\mathrm{BFO}$ nanoislands, although the amount might be very small.

We also determined the crystal structure and epitaxial nature of the BFO nanoislands grown on the STO:Nb (100) substrate. BFO nanoislands were well grown along the (001) orientation, 
(a)

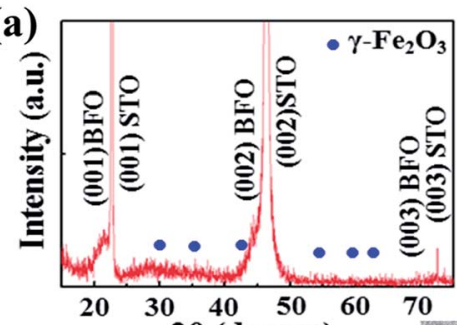

(c)

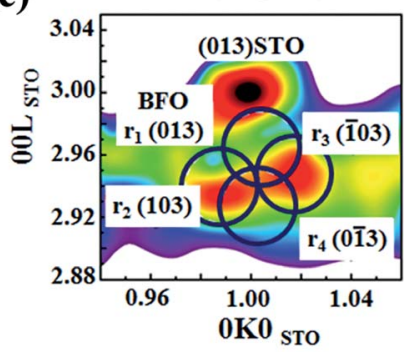

(b)
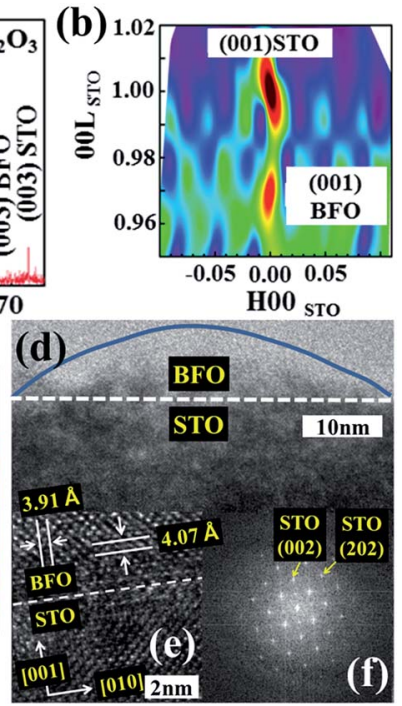

Fig. 5 Epitaxially grown BFO nanoislands on the STO:Nb (100) substrate. (a) $\theta-2 \theta$ scan of nanoislands. The blue dots represent locations of $\gamma-\mathrm{Fe}_{2} \mathrm{O}_{3}$ peaks. RSM image around (b) 001 and (c) 013 STO:Nb reflections for BFO nanoislands. (d) Cross-sectional HR-TEM image of a single BFO nanoisland on STO:Nb (100). (e) Magnified view at the interface between BFO and STO:Nb. (f) SAED pattern obtained from the interface.

which was proved by two-dimensional reciprocal space mapping (RSM) near STO:Nb (001) as shown in Fig. 5b. The domain structure of the BFO nanoislands was also investigated by RSM near the STO:Nb (013) reflection as shown in Fig. $5 \mathrm{c}$. In contrast to the STO:Nb (013) peak, BFO nanoislands showed a broad peak with different shapes.

This peak is roughly divided into four different domains of the rhombohedral phase. ${ }^{26,27}$ Since the STO:Nb substrate has a miscut less than $0.3^{\circ}$, the growth of BFO does not follow the step-flow mode. Thus, a single nanoisland could not have a monodomain crystal, that is, many different polarization directions would exist even in a single nanoisland. The lattice parameter of in-plane and out-of-plane is $3.91 \AA$ and $4.07 \AA$, respectively, as shown by high-resolution transmission electron microscopy (HR-TEM) (Fig. $5 \mathrm{~d}$ and e). This indicates that for $\mathrm{BFO}$ nanoislands in-plane strain is more compressive than outof-plane. ${ }^{26}$ Finally, the epitaxial relationship between the BFO nanoisland and the substrate was confirmed by selected area electron diffraction (SAED) as shown in Fig. $5 f$.

The ferroelectric properties of the BFO nanoislands were investigated by PFM, and the results are given in Fig. 6 . The higher amplitude in the PFM amplitude image corresponds to the higher magnitude of piezoresponse. Thus, the brighter regions show greater piezoresponse as shown in Fig. 6b. Bright regions in the PFM phase image correspond to z-component spontaneous polarization oriented "upward", whereas dark regions represent a polarization component oriented "downward". Since the bright (or dark) regions are not clearly defined in a single nanoisland compared with AFM topography images, complex ferroelectric domains would exist in a single BFO nanoisland as shown in Fig. 6c, which is consistent with RSM results given in Fig. 5c. By
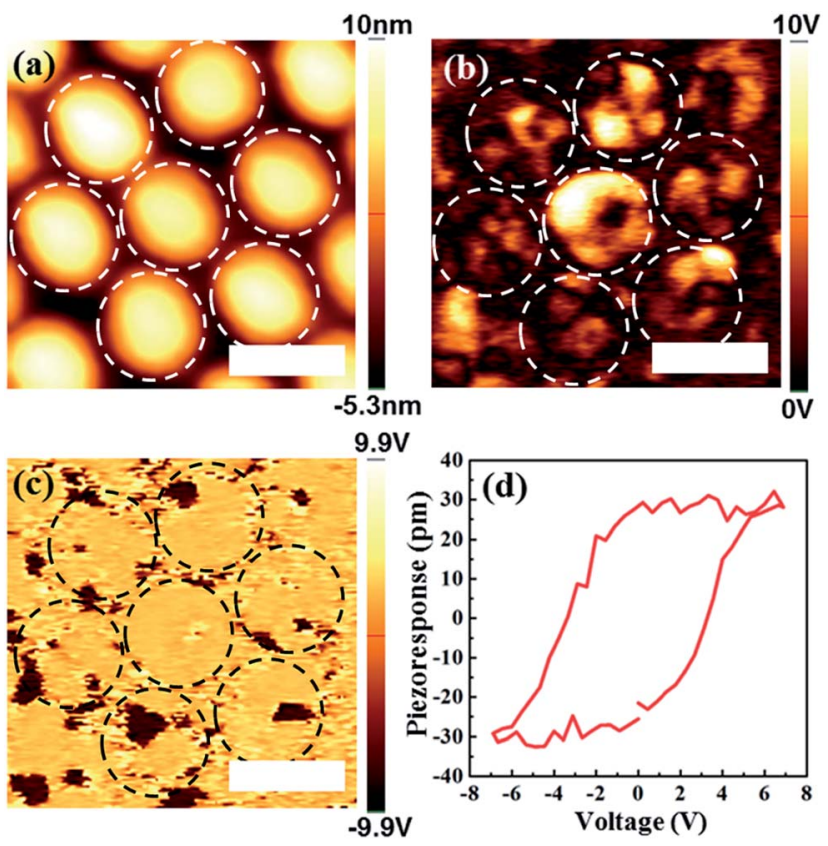

Fig. 6 (a) AFM topography, (b) PFM amplitude and (c) PFM phase images. All of the circles in $(a-c)$ are the same positions of BFO nanoislands. (d) Piezoresponse hysteresis loop of a single BFO nanoisland. The scale bar is $100 \mathrm{~nm}$.

varying the voltage changes from +7 to $-7 \mathrm{~V}$, a hysteresis loop was clearly observed in Fig. 6d.

The magnetic properties of the epitaxially grown $\mathrm{BFO}$ nanoislands were measured by a superconducting quantum interference device (SQUID) based magnetic property measurement system (MPMS) at room temperature along both in-plane [100] and out-of-plane [001] directions as shown in Fig. $7 \mathrm{a}$ and $\mathrm{b}$. The magnetic contribution from the substrate was subtracted from all the data (ESI Fig. S4†). We confirmed that
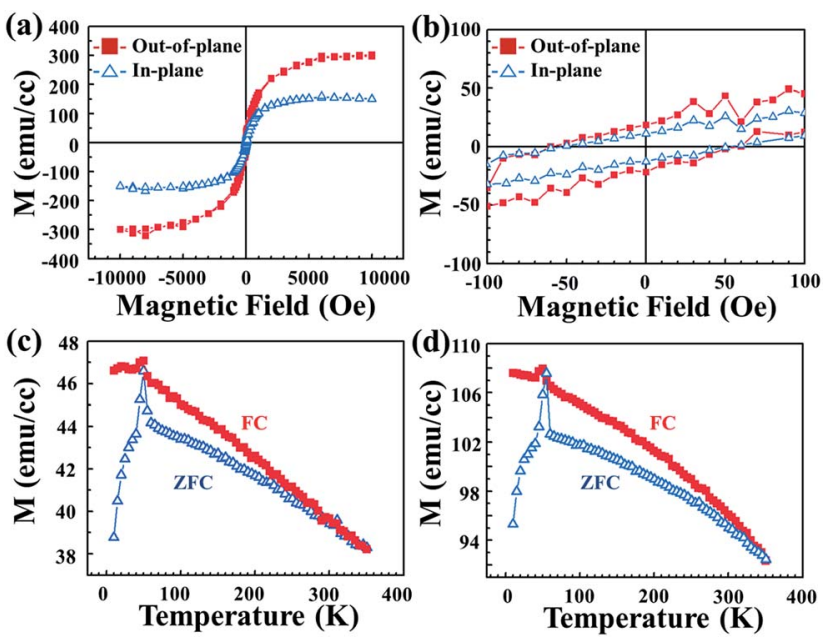

Fig. 7 (a) Magnetic hysteresis loops measured by SQUID based MPMS at room temperature. (b) Magnified view of (a) at a magnetic field from -100 Oe to 100 Oe. (c) In-plane and (d) out-of-plane magnetization of BFO nanoislands as a function of temperature measured under ZFC and 500 Oe FC conditions. 
BFO nanoislands exhibit magnetic property at room temperature from the measurement of temperature dependence of magnetic properties from $10 \mathrm{~K}$ to $350 \mathrm{~K}$ as shown in Fig. $7 \mathrm{c}$ and $\mathrm{d}$.

A clear magnetic hysteresis loop of whole BFO nanoislands is shown in Fig. 7a. Coercive field $\left(H_{\mathrm{c}}\right)$ and remnant magnetization $\left(M_{\mathrm{r}}\right)$ measured along both in plane and out-of-the plane directions are almost the same $\left(H_{\mathrm{c}}=60 \pm 10\right.$ Oe and $\left.M_{\mathrm{r}}=15 \pm 5 \mathrm{emu} \mathrm{cm}^{-3}\right)$, as shown in Fig. 7b. However, the saturation magnetization $\left(M_{\mathrm{s}}\right)$ measured along the in-plane direction was $175 \pm 5 \mathrm{emu} \mathrm{cm} \mathrm{cm}^{-3}$, which is smaller than that $\left(300 \pm 5 \mathrm{emu}^{-3}\right)$ measured along the out-of-plane direction. These $M_{\mathrm{s}}$ are higher than that $(\sim 150$ emu $\mathrm{cm}^{-3}$ for both in-plane and out of plane) of BFO thin film. ${ }^{4} \mathrm{~A}$ higher $M_{\mathrm{S}}$ of BFO nanoislands compared with BFO thin film, especially $M_{\mathrm{S}}$ measured along the out of plane direction, is attributed to the disk-like shape of BFO nanoislands, anisotropy of magneto-crystalline (strained from defects and Nb:STO substrate on the surface), and existence of a second phase. Distinct ferromagnetic properties of BFO nanoislands, compared with bulk BFO which shows negligible (or very weak) ferromagnetic properties are ascribed to the existence of canted antiferromagnetic spins, that is, the antiferromagnetic ordering of BFO is G-type, that is, the nearest neighboring Fe moments are aligned antiparallel to each other in all six $\mathrm{Fe}^{3+}$ spins. But, in reality, those spins are not perfectly antiparallel because of the weak canting moment resulting from local magnetoelectric coupling to the polarization. This phenomenon is observed when the sample size is smaller than the cycloidal length of $64 \mathrm{~nm} .{ }^{28-32}$ Since BFO nanoislands employed in this study have a diameter of $47 \pm 10$ $\mathrm{nm}$ and height of $15 \pm 5 \mathrm{~nm}$, net magnetization could exist. Also, the in-plane lattice parameter of the BFO nanoisland is highly strained by the STO:Nb substrate, as shown in XRD and HR-TEM data (lattice parameter of in-plane and out-of-plane is $3.91 \AA$ and $4.07 \AA$, respectively). However, as described above, since a tiny amount of $\gamma-\mathrm{Fe}_{2} \mathrm{O}_{3}$ might exist, it could contribute to the magnetic response as well.

Fig. $7 \mathrm{c}$ and $\mathrm{d}$ show the magnetization of BFO nanoislands at 500 Oe as a function of temperature after (1) zero-field cooling and (2) 500 Oe field cooling (FC). Both in-plane and out-of-plane curves show the same situation. Magnetization curves measured by ZFC and FC clearly show discontinuity near $55 \mathrm{~K}$. This behavior indicates the co-existence of antiferromagnetic and ferromagnetic properties. ${ }^{29,30}$ Increased magnetization for ZFC $\left(M_{\mathrm{ZFC}}\right)$ with decreasing temperature from 350 to $50 \mathrm{~K}$ is induced by the ferromagnetic behavior of BFO nanoislands. The competition between magnetic anisotropy energy and thermal energy results in the existence of the broad peak in $M_{\mathrm{ZFC}}$ curves. The sharp cusp presented around $55 \mathrm{~K}\left(T_{\text {cusp }}\right)$ in ZFC and FC curves is attributed to the magnetic domain pinning effect.

\section{Conclusions}

We have successfully fabricated a high density array of multiferroic BFO nanoislands in a large area by using a facile and innovative method to complement the limitation of existing fabrication methods of AAO mask-assisted nanoisland array. The high density array of BFO nanoislands in a large area showed both ferroelectricity of individual nanoislands and macroscopic magnetism, that is, multiferroicity, at room temperature. The array also shows hysteresis loops for both ferroelectric and magnetic properties. Especially, saturation magnetization of BFO nanoislands is higher than that of thin film. This is mainly ascribed to the existence of canted antiferromagnetic spins due to the small size of BFO nanoislands, highly strained BFO nanoislands, and/or existence of a second phase, for instance, $\gamma-\mathrm{Fe}_{2} \mathrm{O}_{3}$. A high density array of multiferroic nanoislands in a large area could be employed as the next generation memory device capable of electric writing and magnetic reading (or vice versa).

\section{Acknowledgements}

This work was supported by the National Creative Research Initiative Program supported by the National Research Foundation of Korea (NRF) grant (no. 2013R1A3A2042196) funded by the Korean government (MEST). It was also supported by the Basic Science Research program through NRF funded by the Ministry of Science, ICT \& Future Planning (NRF2014R1A4A1008474).

\section{References}

1 G. Catalan and J. F. Scott, Adv. Mater., 2009, 21, 2463.

2 S. H. Baek, H. W. Jang, C. M. Folkman, Y. L. Li, B. Winchester, J. X. Zhang, Q. He, Y. H. Chu, C. T. Nelson, M. S. Rzchowski, X. Q. Pan, R. Ramesh, L. Q. Chen and C. B. Eom, Nat. Mater., 2010, 9, 309.

3 J. F. Scott, J. Mater. Chem., 2012, 22, 4567.

4 J. Wang, J. B. Neaton, H. Zheng, V. Nagarajan, S. B. Ogale, B. Liu, D. Viehland, V. Vaithyanathan, D. G. Schlom, U. V. Waghmare, N. A. Spaldin, K. M. Rabe, M. Wuttig and R. Ramesh, Science, 2003, 229, 1719.

5 T. Zhao, A. Scholl, F. Zavaliche, K. Lee, M. Barry, A. Doran, M. P. Cruz, Y. H. Chu, C. Ederer, N. A. Spaldin, R. R. Das, D. M. Kim, S. H. Baek, C. B. Eom and R. Ramesh, Nat. Mater., 2006, 5, 823.

6 Y. Y. Liu, R. K. Vasudevan, K. Pan, S. H. Xie, W.-I. Liang, A. Kumar, S. Jesse, Y.-C. Chen, Y.-H. Chu, V. Nagarajan, S. V. Kalinine and J. Y. Li, Nanoscale, 2012, 4, 3175.

7 J. X. Zhang, R. J. Zeches, Q. He, Y.-H. Chuce and R. Ramesh, Nanoscale, 2012, 4, 6196.

8 I. Vrejoiu, M. Alexe, D. Hesse and U. Gösele, Adv. Funct. Mater., 2008, 18, 3892.

9 T. Sun, Z. Pan, V. P. Dravid, Z. Wang, M. F. Yu and J. Wang, Appl. Phys. Lett., 2006, 89, 163117.

10 I. Jung and J. Y. Son, J. Am. Ceram. Soc., 2012, 95, 3716. 11 W.-H. Kim and J. Y. Son, Appl. Phys. Lett., 2013, 103, 052905. 12 Y. Kim, H. Han, Y. Kim, W. Lee, M. Alexe, S. Baik and J. K. Kim, Nano Lett., 2010, 10, 2141.

13 Y. Kim, Y. Kim, H. Han, S. Jesse, S. Hyun, W. Lee, S. V. Kalinin and J. K. Kim, J. Mater. Chem. C, 2013, 1, 5299. 14 H. Zheng, F. Straub, Q. Zhan, P.-L. Yang, W.-K. Hsieh, F. Zavaliche, Y.-H. Chu, U. Dahmen and R. Ramesh, Adv. Mater., 2006, 18, 2747. 
15 A. Morelli, F. Johann, N. Schammelt, D. McGrouther and I. Vrejoiu, J. Appl. Phys., 2013, 113, 154101.

16 S. Hong, T. Choi, J. H. Jeon, Y. Kim, H. Lee, H. Y. Joo, I. Hwang, J. S. Kim, S. O. Kang, S. V. Kalinin and B. H. Park, Adv. Mater., 2013, 25, 2339.

17 S.-H. Lyu and J.-S. Lee, J. Mater. Chem., 2012, 22, 1852.

$18 \mathrm{~J}$. Varghese, R. W. Whatmoreb and J. D. Holmes, J. Mater. Chem. C, 2013, 1, 2618.

19 X. Gao, L. Liu, B. Birajdar, M. Ziese, W. Lee, M. Alexe and D. Hesse, Adv. Funct. Mater., 2009, 19, 3450.

20 S. Y. Yang, G. Jeon and J. K. Kim, J. Mater. Chem., 2012, 22, 23017.

21 G. Koster, B. L. Kropman, G. J. H. M. Rijnders, D. H. A. Blank and H. Rogalla, Appl. Phys. Lett., 1998, 73, 2920.

22 J. Chen, X. Xing, A. Watson, W. Wang, R. Yu, J. Deng, L. Yan, C. Sun and X. Chen, Chem. Mater., 2007, 19, 3598.

23 D. Kothari, V. R. Reddy and A. Gupta, AIP Conf. Proc., 2010, 1313, 212.

24 R. Nechache, C. V. Cojocaru, C. Harnagea, C. Nauenheim, M. Nicklaus, A. Ruediger, F. Rosei and A. Pignolet, $A d v$. Mater., 2011, 23, 1724.
25 Z. Li, Y. Shen, Y. Guan, Y. Hu, Y. Lin and C.-W. Nan, J. Mater. Chem. A, 2014, 2, 1967.

26 H. W. Jang, D. Ortiz, S.-H. Baek, C. M. Folkman, R. R. Das, P. Shafer, Y. Chen, C. T. Nelson, X. Pan, R. Ramesh and C. B. Eom, Adv. Mater., 2009, 21, 817.

27 S. K. Streiffer, C. B. Parker, A. E. Romanov, M. J. Lefevre, L. Zhao, J. S. Speck, W. Pompe, C. M. Foster and G. R. Bai, J. Appl. Phys., 1998, 83, 2742.

28 S. Ryu, J. Y. Kim, Y. H. Shin, B. G. Park, J. Y. Son and H. M. Jang, Chem. Mater., 2009, 21, 5050.

29 S. M. Selbach, T. Tybell, M.-A. Einarsrud and T. Grande, Chem. Mater., 2007, 19, 6478.

30 T.-J. Park, G. C. Papaefthymiou, A. J. Viescas, A. R. Moodenbaugh and S. S. Wong, Nano Lett., 2007, 7, 766. 31 F. Huang, Z. Wang, X. Lu, J. Zhang, K. Min, W. Lin, R. Ti, T. Xu, J. He, C. Yue and J. Zhu, Sci. Rep., 2013, 3, 2907.

32 M. M. Shirolkar, C. Hao, X. Dong, T. Guo, L. Zhang, M. Li and H. Wang, Nanoscale, 2014, 6, 4735. 